\title{
Molecular epidemiology of canine parvovirus type 2 in Italy from 1994 to 2017: recurrence of the CPV-2b variant
}

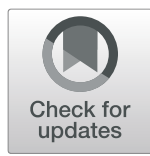

Mara Battilani ${ }^{1 *}$ D, Francesco Modugno ${ }^{2}$, Francesco Mira², Giuseppa Purpari², Santina Di Bella², Annalisa Guercio ${ }^{2}$ and Andrea Balboni ${ }^{1}$

\begin{abstract}
Background: Canine parvovirus type 2 (CPV-2) is the most important enteric virus infecting canids. It is a rapidly evolving virus; after its emergence in the 1970s, new antigenic variants (called CPV-2a, 2b and 2c) emerged and replaced the original antigenic type. The three antigenic variants are globally distributed with different frequencies and levels of genetic variability. This study focused on VP2 gene sequence analysis and the phylodynamics of CPV-2 which were detected in 123 dogs showing clinical signs of gastroenteritis collected in Italy from 1994 to 2017.

Results: For the most part, the sick dogs were young, and a third of them (32.5\%) had been vaccinated. No statistical association was found between the CPV-2 antigenic variants, and sex, age, breed and vaccination status. Sequence analysis showed that all three antigenic types circulated in Italy; the CPV-2a type was the prominent genotype, followed by CPV-2c and CPV-2b, with notable differences regarding regional bases and significant fluctuations over time. Nucleotide sequence data showed high genetic heterogeneity with 67 nucleotide sequence types (ntSTs) identified, corresponding to 21 amino acid sequence types (aaSTs). The aaSTs and ntSTs obtained were distributed differently among the three CPV-2 antigenic variants: CPV-2a grouped 12/21 (57.1\%) aaSTs and 41/67 (61.2\%) ntSTs; CPV2b grouped 5/21 (23.8\%) aaSTs and 6/67 (8.9\%) ntSTs, and CPV-2c grouped 4/21 (19.1\%) aaSTs and 20/67 (29.9\%) ntSTs. Canine parvovirus $2 \mathrm{a}$ was characterised by the highest genetic variability while CPV-2c was characterised by notable stability with a predominant amino acid profile during the entire sampling time. Canine parvovirus $2 \mathrm{~b}$ re-emerged in recent years, showing a new and distinctive amino acid profile of the VP2 protein.

Conclusions: The findings of the present study provided new insights regarding the phylodynamics and evolution of CPV-2 in Italy, pointing out notable differences at the local level in the distribution of the CPV-2 variants and the selection of genetic subtypes. The evolution of CPV-2 has raised questions regarding the efficacy of vaccination; therefore, continuous monitoring regarding the evolution and spread of new CPV-2 variants should be a key aim of ongoing research.
\end{abstract}

Keywords: Canine parvovirus, Dog, Epidemiology, Evolution, Phylodynamics

\section{Background}

Canine parvovirus type $2(\mathrm{CPV}-2)$ is a non-enveloped single-stranded DNA virus belonging to the family Parvoviridae, the subfamily Parvovirinae and the genus Protoparvovirus according to the International Committee on Taxonomy of Viruses (ICTV) [1]. Recently,

\footnotetext{
* Correspondence: mara.battilani@unibo.it

'Department of Veterinary Medical Sciences, Alma Mater Studiorum -

University of Bologna, Via Tolara di Sopra 50, 40064 Ozzano dell'Emilia, BO, Italy

Full list of author information is available at the end of the article
}

CPV-2 has been included within the species Carnivore protoparvovirus 1 which also includes other closely related autonomous viruses causing a range of serious conditions, especially in young animals: feline panleukopenia virus (FPV, the prototype virus of the former carnivore protoparvovirus), mink enteritis virus (MEV) and raccoon parvovirus (RaPV). These viruses are considered to be host variants of a unique viral species, given the reciprocal high genomic and antigenic relationship [2].

(c) The Author(s). 2019 Open Access This article is distributed under the terms of the Creative Commons Attribution 4.0 International License (http://creativecommons.org/licenses/by/4.0/), which permits unrestricted use, distribution, and reproduction in any medium, provided you give appropriate credit to the original author(s) and the source, provide a link to the Creative Commons license, and indicate if changes were made. The Creative Commons Public Domain Dedication waiver (http://creativecommons.org/publicdomain/zero/1.0/) applies to the data made available in this article, unless otherwise stated. 
Canine parvovirus type 2 is responsible for acute gastroenteritis in dogs, often fatal in 6-12-week-old puppies. In fact, despite vaccination, it is still widespread in the canine population and, if pups are not vaccinated or when maternal antibodies interfere with their vaccination, they generally become naturally infected [3]. Furthermore, CPV-2 infection has also been reported in vaccinated adult dogs [4].

Although CPV-2 is a DNA virus, its genomic substitution rate is similar to RNA viruses, with a value of approximately $10^{-4}$ substitutions per site per year [5]. Consequently, after its emergence in the late 1970s, CPV2 has been undergoing rapid evolution and, in just a few years, the original antigenic type 2 has been completely replaced by the new antigenic variants called CPV-2a, $-2 b$ and $-2 c$, based on key amino acid substitutions in the VP2 protein [6,7]. These amino acid changes have provided important biological properties and have enabled the CPV-2 variants to replicate and spread more effectively in susceptible hosts. In fact, CPV-2a, $2 \mathrm{~b}$ and $2 \mathrm{c}$ have reappeared in the host range for cats [8] and have increased their own pathogenicity, causing more severe disease with a shorter incubation period; moreover, the new virus types are shed in the faeces at much higher titres, and a lower virus dose seems to be required for efficient infection [9].

Currently, the original antigenic type 2 is present only in commercial vaccines, and the virus types $2 a, 2 b$ and $2 \mathrm{c}$ are variously distributed in the canine population worldwide. Numerous scientific papers have reported the frequencies of the different CPV-2 variants in several geographic areas [10]. Epidemiological surveys regarding the distribution of the CPV-2 variants in different countries have shown that CPV-2a is the predominant variant in most of Asia and in European countries, and is the only variant reported in New Zealand. The CPV-2b variant was found to be the predominant antigenic variant in Ireland, the UK, the U.S.A., African countries, several Asian countries and Australia [11]. The CPV-2c variant has mainly been found in European countries and South America, and it has recently been detected in the Australian dog population [12].

In Italy, CPV-2a appeared to be the predominant variant maintaining its prevalence on the others over the time [13-15]. In recent decades, a nearly complete substitution of CPV-2b by CPV-2c has been observed $[9,16]$ although, despite the initial and sudden peak of detections [17], $\mathrm{CPV}-2 \mathrm{c}$ was the least frequently sequenced variant during the period of the study. A notable difference at the level of local geographic areas has been observed in the distribution of the CPV-2b variant in Italy, with its absence in Sicily [18], and its dominant prevalence in Sardinia [19].

The typing of the CPV-2 variants is commonly based on the different amino acids observed in residue 426 of the VP2 protein (Asn in CPV-2a, Asp in CPV-2b and
Glu in CPV-2c), although other specific amino acid changes in VP2 residues have been observed. The CPV$2 \mathrm{a}$ and CPV-2b variants showing amino acid change 297 Ser $\rightarrow$ Ala have been designated as the "new CPV-2a" and "new CPV-2b" [20, 21]; viruses showing a 300 $\mathrm{Gly} \rightarrow$ Asp mutation were designated as "CPV-2c(a)" and "CPV-2(b)" [22]. The Italian CPV-2b variants from Sardinia contained additional amino acid substitutions, and $371 \mathrm{Ala} \rightarrow$ Gly and $418 \mathrm{Ile} \rightarrow$ Thr were named the "new CPV-2b" [19]. Along with other specific changes, such as $440 \mathrm{Thr} \rightarrow \mathrm{Ala}$ and $324 \mathrm{Tyr} \rightarrow \mathrm{Ile}, \mathrm{CPV}-2$ did not receive any clear taxonomy or common designation despite their global presence. Other authors have proposed that, in the case of the presence of a new site mutation, the virus could be added into sustainable nomenclature as a new sub-variant [23]. Although not all scientists are in agreement with the virus nomenclature which is somewhat confusing, references to CPV-2a, 2b and $2 \mathrm{c}$ are prevalent in the literature. Some of these mutations have changed the antigenic properties of the capsid and have reached high frequencies in viral populations, but the biological consequences of the majority of these changes are, for the most part, unknown [24]. The CPV-2 evolution is ongoing, but the dynamics of the spread and the variation of the virus have recently changed. While the emergence of CPV-2 has been characterised by global viral spread, the endemic phase of the disease has been characterised by geographical genetic differentiation [25] with CPV-2 capsid variants which appear to circulate predominantly locally [26]. The evolution of this virus has raised questions regarding the efficacy of some vaccines; therefore, an understanding of the variation is required.

This study focused on VP2 sequence analysis and the phylodynamics of CPV-2 which were detected in dogs showing clinical signs of gastroenteritis collected in Italy from 1994 to 2017.

\section{Results \\ Study group}

The signalment data of the 123 dogs included in this study which tested positive for CPV-2 DNA are summarised in Table 1 . The median age of the dogs was 3 months (range 1 month -10 years). The dogs were of different breeds and of both sexes. Ninety-four out of the $123(76.4 \%)$ dogs came from the mainland of Northern and Central Italy (only two came from Southern Italy, the Basilicata and Apulia regions, respectively) and 29/123 (23.6\%) dogs were sampled in the insular area of Italy (the island of Sicily in Southern Italy) in 2009-2010. Forty out of the $123(32.5 \%)$ dogs were vaccinated whereas $57 / 123$ (46.3\%) dogs were unvaccinated or incompletely vaccinated; the vaccination status of the remaining 26/123 (21.1\%) dogs was unknown. 
Table 1 Descriptive statistics of the dogs included in the study population

\begin{tabular}{|c|c|c|c|c|c|}
\hline & $\begin{array}{l}\text { Dogs positive to } \\
\text { CPV-2 DNA } \\
n=123(\%)\end{array}$ & $\begin{array}{l}\text { CPV-2a } \\
n=56(\%)\end{array}$ & $\begin{array}{l}\text { CPV-2b } \\
n=18(\%)\end{array}$ & $\begin{array}{l}\text { CPV-2C } \\
n=49(\%)\end{array}$ & $P$ value \\
\hline \multicolumn{6}{|l|}{ Year of sampling } \\
\hline 1994 & $1(0.8)$ & $1(1.8)$ & 0 & 0 & \multirow[t]{23}{*}{$<0.0001$} \\
\hline 1995 & $5(4.1)$ & $2(3.6)$ & $3(16.7)$ & 0 & \\
\hline 1996 & $2(1.6)$ & $1(1.8)$ & $1(5.6)$ & 0 & \\
\hline 1997 & $1(0.8)$ & $1(1.8)$ & 0 & 0 & \\
\hline 1998 & $2(1.6)$ & $2(3.6)$ & 0 & 0 & \\
\hline 1999 & $4(3.3)$ & $4(7.1)$ & 0 & 0 & \\
\hline 2000 & $7(5.7)$ & $6(10.7)$ & 0 & $1(2)$ & \\
\hline 2001 & $4(3.3)$ & $3(5.4)$ & 0 & $1(2)$ & \\
\hline 2002 & $2(1.6)$ & $2(3.6)$ & 0 & 0 & \\
\hline 2003 & $1(0.8)$ & $1(1.8)$ & 0 & 0 & \\
\hline 2005 & $1(0.8)$ & $1(1.8)$ & 0 & 0 & \\
\hline 2006 & $4(3.3)$ & $2(3.6)$ & 0 & $2(4.1)$ & \\
\hline 2007 & $5(4.1)$ & $3(5.4)$ & 0 & $2(4.1)$ & \\
\hline 2008 & $8(6.5)$ & $7(12.5)$ & 0 & $1(2)$ & \\
\hline 2009 & $37(30.1)$ & $8(14.3)$ & $3(16.7)$ & $26(53.1)$ & \\
\hline 2010 & $7(5.7)$ & $3(5.4)$ & $3(16.7)$ & $1(2)$ & \\
\hline 2011 & $4(3.3)$ & $4(7.1)$ & 0 & 0 & \\
\hline 2012 & $10(8.1)$ & $3(5.4)$ & 0 & $7(14.3)$ & \\
\hline 2013 & $4(3.3)$ & 0 & $1(5.6)$ & $3(6.1)$ & \\
\hline 2014 & $4(3.3)$ & $2(3.6)$ & 0 & $2(4.1)$ & \\
\hline 2015 & $2(1.6)$ & 0 & $1(5.6)$ & $1(2)$ & \\
\hline 2016 & $4(3.3)$ & 0 & $3(16.7)$ & $1(2)$ & \\
\hline 2017 & $4(3.3)$ & 0 & $3(16.7)$ & $1(2)$ & \\
\hline \multicolumn{6}{|l|}{ Sex } \\
\hline Male & 61 (49.6) & $26(46.4)$ & $9(50)$ & $26(53.1)$ & \multirow[t]{3}{*}{0.7866} \\
\hline Female & $37(30.1)$ & $18(32.2)$ & $4(22.2)$ & 15 (30.6) & \\
\hline NA & $25(20.3)$ & $12(21.4)$ & $5(27.8)$ & $8(16.4)$ & \\
\hline \multicolumn{6}{|l|}{ Age } \\
\hline$<1$ & $102(82.9)$ & $46(82.1)$ & $15(83.3)$ & $41(83.7)$ & \multirow[t]{4}{*}{0.1496} \\
\hline $1-5$ & $4(3.3)$ & $3(5.4)$ & 0 & $1(2)$ & \\
\hline$\geq 6$ & $3(2.4)$ & $2(3.6)$ & $1(5.6)$ & 0 & \\
\hline NA & $14(11.4)$ & $5(8.9)$ & $2(11.1)$ & $7(14.3)$ & \\
\hline \multicolumn{6}{|l|}{ Breed } \\
\hline Pure breeds & $74(60.2)$ & $37(66.1)$ & $12(66.6)$ & $25(51)$ & \multirow[t]{3}{*}{0.3228} \\
\hline Mixed & $35(28.4)$ & $13(23.2)$ & $5(27.8)$ & $17(34.7)$ & \\
\hline NA & $14(11.4)$ & $6(10.7)$ & $1(5.6)$ & $7(14.3)$ & \\
\hline \multicolumn{6}{|l|}{ Origin } \\
\hline Piedmont & $1(0.8)$ & 0 & $1(5.6)$ & 0 & \multirow[t]{5}{*}{0.0001} \\
\hline Veneto & $4(3.3)$ & $4(7.1)$ & 0 & 0 & \\
\hline Friuli-Venezia Giulia & $1(0.8)$ & 0 & $1(5.6)$ & 0 & \\
\hline Emilia Romagna & $80(65.0)$ & $42(75)$ & $15(83.3)$ & $23(46.9)$ & \\
\hline Tuscany & $3(2.4)$ & $2(3.6)$ & $1(5.6)$ & 0 & \\
\hline
\end{tabular}


Table 1 Descriptive statistics of the dogs included in the study population (Continued)

\begin{tabular}{lllll}
\hline & $\begin{array}{l}\text { Dogs positive to } \\
\text { CPV-2 DNA } \\
n=123(\%)\end{array}$ & $\begin{array}{l}\text { CPV-2a } \\
n=56(\%)\end{array}$ & $\begin{array}{l}\text { CPV-2b } \\
n=18(\%)\end{array}$ & $\begin{array}{l}\text { CPV-2C } \\
n=49(\%)\end{array}$ \\
\hline Lazio & $1(0.8)$ & $1(1.8)$ & 0 & 0 \\
Marche & $1(0.8)$ & 0 & 0 & 0 \\
Abruzzo & $1(0.8)$ & $1(1.8)$ & 0 & 0 \\
Basilicata & $1(0.8)$ & $1(1.8)$ & 0 & $1(2)$ \\
Apulia & $1(0.8)$ & 0 & 0 & $24(49)$ \\
Sicily & $29(23.6)$ & $5(8.9)$ & 0 & $14(28.6)$ \\
Vaccination & & $21(37.5)$ & $5(27.8)$ & $1(2)$ \\
Completely vaccinated & $40(32,5)$ & $2(3.6)$ & $1(5.6)$ & $21(42.9)$ \\
Incompletely vaccinated & $4(3.3)$ & $23(41.1)$ & $9(50)$ & $13(26.5)$ \\
Unvaccinated & $53(43.1)$ & $10(17.8)$ & $3(16.6)$ & 0.6799 \\
NA & $26(21.1)$ & & & 0 \\
\hline
\end{tabular}

Data are given as number of dogs and (\% of dogs on the total number of dogs included in the group). The data not available (in grey) were not statistically analysed. Significance was set at $P<0.05$. n: total number of dogs included in the study group. NA data not available

\section{Typing of CPV-2 and DNA sequence analysis}

In order to characterise the CPV-2 detected, the coding VP2 gene sequence of 1745 nts was obtained from all viruses. Based on the 426 amino acid residues of the deduced VP2 protein, 56/123 (45.5\%) CPV-2 s were classified as the $2 \mathrm{a}$ variant owing to the presence of the AAT codon which codes for the amino acid asparagine, $18 / 123$ (14.6\%) viruses belonged to CPV-2b owing to the presence of the amino acid aspartate (codon GAT) and 49/123 (39.8\%) were characterised as CPV-2c owing to the occurrence of the amino acid glutamate (codon GAA). The original CPV type 2 was not found.

Nucleotide sequences from the 123 viruses sequenced showed high genetic heterogeneity with 67 nucleotide sequence types (ntSTs) (Additional file 1). The majority $(46 / 67,68.7 \%)$ of the ntSTs grouped one nucleotide sequence while 17/67 (25.4\%) ntSTs grouped from 2 to 4 nucleotide sequences. The remaining four ntSTs $(4 / 67$, 6\%), called ntST04 (CPV-2a), ntST38 (CPV-2b), ntST40 (CPV-2c) and ntST43 (CPV-2c), grouped 8, 11, 11 and 7 nucleotide sequences, respectively.

The VP2 nucleotide sequences were translated into the corresponding amino acid sequences, obtaining 21 amino acid sequence types (aaSTs) (Additional file 1).
Five out of the $21(23.8 \%)$ aaSTs included 104/123 (84.5\%) nucleotide sequences corresponding to 51/67 (76.1\%) ntSTs (Table 2). The remaining 16/21 (76.2\%) aaSTs included 19/123 (15.5\%) nucleotide sequences corresponding to only one ntST each $(16 / 67 \mathrm{ntSTs}$, 23.9\%). The aaSTs and ntSTs obtained in this study were distributed differently in the three CPV-2 antigenic variants (Additional file 1): CPV-2a grouped 12/21 (57.1\%) aaSTs and 41/67 (61.2\%) ntSTs; CPV-2b grouped 5/21 (23.8\%) aaSTs and 6/67 (8.9\%) ntSTs and CPV-2c grouped 4/21 (19.1\%) aaSTs and 20/67 (29.9\%) ntSTs (Fig. 1).

Regarding the 29 viral strains collected in Sicily, 28 were sampled in 2009 and one in 2010. Five out of the 29 (17.2\%) viral sequences were CPV-2a and were distinguishable in five ntSTs; the remaining $24 / 29$ (82.8\%) sequences were typed as CPV-2c and were distinguishable in six ntSTs (comprising ntST40 and ntST43), prevalently grouped in aaST06 (Additional file 1). No CPV-2b was detected in Sicily. Only one ntST grouping of the Sicilian viral sequences (CPV-2c ntST43) also contained mainland viral sequences (134/2012 KF373602 and 138/ 2012 MK348086); the remaining ntSTs grouped only Sicilian sequences.

Table 2 Main amino acid sequence types (aaSTs)

\begin{tabular}{llll}
\hline & Antigenic variant & Number $^{\mathrm{a}}$ and $(\%)$ of ntSTs & Number $^{\mathrm{b}}$ and (\%) of nucleotide sequences \\
\hline aaST01 & CPV-2a & $25 / 67(37.3 \%)$ & $39 / 123(31.7 \%)$ \\
aaST06 & CPV-2C & $17 / 67(25.4 \%)$ & $44 / 123(35.8 \%)$ \\
aaST07 & CPV-2a & $5 / 67(7.5 \%)$ & $6 / 123(4.9 \%)$ \\
aaST11 & CPV-2a & $2 / 67(3 \%)$ & $2 / 123(1.6 \%)$ \\
aaST14 & CPV-2b & $2 / 67(3 \%)$ & $13 / 123(10.6 \%)$ \\
\hline
\end{tabular}

aaST amino acid sequence type, CPV-2 canine parvovirus type $2, n t S T$ nucleotide sequence type

${ }^{a}$ Expressed as number of nucleotide sequence types (ntSTs) included in the aaST on the total number of ntSTs identified in this study

${ }^{b}$ Expressed as number of nucleotide sequences included in the aaST on the total number of nucleotide sequences identified in this study 


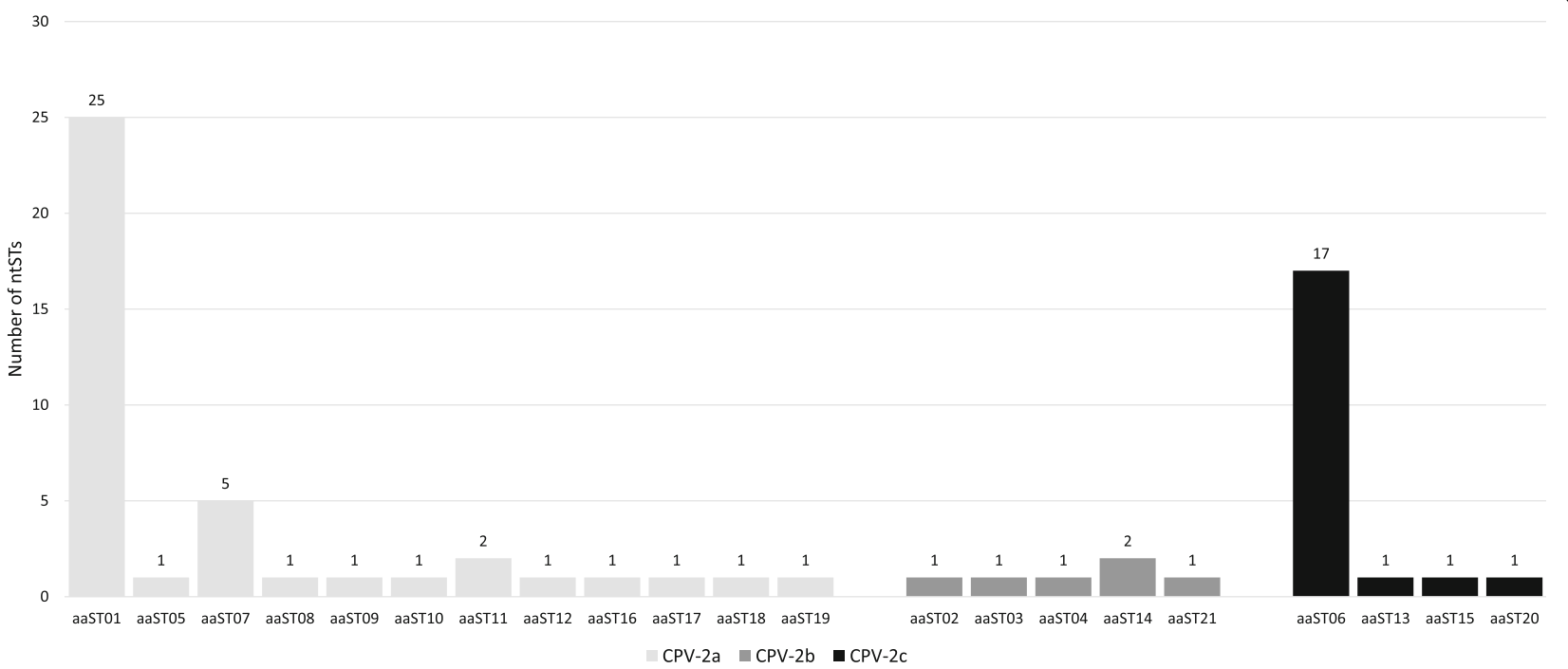

Fig. 1 Distribution of amino acid sequence types and nucleotide sequence types in each CPV-2 antigenic variant. aaST: amino acid sequence type. CPV-2: canine parvovirus type 2 . ntST: nucleotide sequence type

The sequences obtained were aligned and compared to worldwide non-Italian CPV-2 reference strains retrieved from the GenBank database. Nucleotide identity was 99.2-99.9\% among the ntSTs obtained, and $98.6-100 \%$ among the ntSTs and the worldwide non-Italian CPV-2 reference sequences. Amino acidic similarity was 98.999.8\% among the aaSTs obtained in this study and 97.9$100 \%$ among the aaSTs obtained and the worldwide non-Italian CPV-2 reference sequences.

Several synonymous and non-synonymous substitutions were detected by comparing the sequences. The predicted amino acid substitutions are summarised in Table 3. Seventeen non-synonymous changes were detected in the 21 aaSTs other than the amino acid changes in residue 426 which differentiated the antigenic variants. At least six mutations were very common since they were shared by reference strains detected in various parts of the world: residues 13 (Pro $\rightarrow$ Ser), 139 (Val $\rightarrow$ Ile), 297 (Ser $\rightarrow$ Ala), 324 (Tyr $\rightarrow$ Ile), $418 \quad$ (Ile $\rightarrow$ Thr $)$ and 440 ( $\mathrm{Thr} \rightarrow$ Ala). Residue 440 of aaST16, which included the Sicilian CPV-2a X83090/2009 (GenBank ID: KF385388), also showed the change Thr $\rightarrow$ Ser. Other coding changes have already been described but were less common, such as the $265 \mathrm{Thr} \rightarrow$ Pro, $371 \mathrm{Ala} \rightarrow$ Gly, $429 \mathrm{Val} \rightarrow$ Ile, 517 Asn $\rightarrow$ Ser and 568 Gly $\rightarrow$ Ala mutations [13, 19, 27-29]. Instead, the mutations at residues 16 (Arg $\rightarrow$ Lys), 67 (Arg $\rightarrow$ Lys), 75 (Glu $\rightarrow$ Lys), 144 (Glu $\rightarrow$ Lys) and 356 (Pro $\rightarrow$ His) were unique and characteristic of the CPV-2a and CPV-2c variants identified in this study. All the aaSTs characterised as the CPV-2a variant in this study showed the amino acid alanine in residue 297 which is considered a sort of marker of the more recent types $2 \mathrm{a}$ and $2 \mathrm{~b}$ ("new CPV-2a" and "new
CPV-2b") [20, 21]. Instead, only the aaSTs 02,14 and 21 belonging to the CPV-2b variant showed 297Ala while the remaining CPV-2b aaSTs (aaST03 and aaST04) showed the amino acid serine in residue 297. Of the CPV-2b aaSTs, the most recent aaST14 and aaST21 showed unique and distinct mutations with respect to aaST02, aaST03 and aaST04 which were detected in the 1990s: In addition to $297 \mathrm{Ser} \rightarrow \mathrm{Ala}$, aaST14 and aaST21 showed the mutations 371 $\mathrm{Ala} \rightarrow$ Gly and $418 \mathrm{Ile} \rightarrow \mathrm{Thr}$, already described in the Italian strains from Sardinia [19].

The results of the DnaSP analyses and the Simpson diversity index (D) value calculated for each CPV-2 antigenic variant are reported in Table 4. As compared to the other variants, CPV-2a showed the highest sequence variability, assessed by the nucleotide diversity $\mathrm{Pi}(\pi)$ and the average number of nucleotide differences $(\mathrm{k})$. Conversely, the CPV-2c variant showed the lowest sequence variability; the same result was obtained for the CPV-2c variant when analysing only the sequences coming from Sicily as well analysing the type $2 \mathrm{c}$ sequence data set, excluding the Sicilian ones (Additional file 2). The CPV-2a and $2 \mathrm{~b}$ types had the lowest and the highest values of $\mathrm{D}$, respectively, but $C P V-2 b$ showed the highest $a / \eta$ rate (the total number of non-synonymous differences on the total number of mutations), demonstrating a higher impact of nucleotide variability on the viral phenotype.

The CPV-2a, CPV-2b and CPV-2c variants formed three well-distinguishable clusters in the amino acid phylogenetic tree constructed with the VP2 sequences obtained in this study (Fig. 2). However, in the phylogenetic tree constructed with the VP2 nucleotide sequences, the CPV-2c viruses formed a separate cluster whereas 
Table 3 Amino acid sequence types (aaSTs) of the Italian CPV strains

\begin{tabular}{|c|c|c|c|c|c|c|c|c|c|c|c|c|c|c|c|c|c|c|}
\hline aaST of the CPV & 13 & 16 & 67 & 75 & 139 & 144 & 265 & 297 & 324 & 356 & 371 & 418 & 426 & 429 & 440 & 517 & 568 & TYPE \\
\hline aaST01 $(n=39)$ & Pro & $\operatorname{Arg}$ & Arg & Glu & Val & Glu & Thr & Ala & Tyr & Pro & Ala & Ile & Asn & Val & Thr & Asn & Gly & CPV-2a \\
\hline $\operatorname{aaST02}(n=1)$ & - & - & - & - & - & - & - & - & - & - & - & - & Asp & - & - & - & - & CPV-2b \\
\hline aaST03 $(n=2)$ & - & - & - & - & - & - & Pro & Ser & - & - & - & - & Asp & - & - & - & - & CPV-2b \\
\hline aaST04 $(n=1)$ & - & - & - & - & - & - & - & Ser & - & - & - & - & Asp & - & - & - & - & CPV-2b \\
\hline aaST05 $(n=1)$ & - & - & - & - & - & - & - & - & - & - & - & - & - & - & - & Ser & - & CPV-2a \\
\hline aaST06 $(n=44)$ & - & - & - & - & - & - & - & - & - & - & - & - & Glu & - & - & - & - & CPV-2C \\
\hline aaST07 $(n=6)$ & - & - & - & - & - & - & - & - & - & - & - & - & - & - & Ala & - & - & CPV-2a \\
\hline aaST08 $(n=1)$ & - & - & - & - & - & - & - & - & - & - & - & - & - & - & - & - & Ala & CPV-2a \\
\hline aaST09 $(n=1)$ & - & - & Lys & Lys & - & - & - & - & - & - & - & - & - & - & - & - & - & CPV-2a \\
\hline aaST10 $(n=1)$ & - & Lys & - & - & - & Lys & - & - & - & - & - & - & - & - & - & - & - & CPV-2a \\
\hline aaST11 $(n=2)$ & - & Lys & - & Lys & - & - & - & - & - & - & - & - & - & - & - & - & - & CPV-2a \\
\hline aaST12 $(n=1)$ & Ser & - & - & - & - & - & - & - & - & - & - & - & - & - & - & - & - & CPV-2a \\
\hline $\operatorname{aaST13}(n=1)$ & - & - & - & Lys & - & - & - & - & - & - & - & - & Glu & - & - & - & - & CPV-2C \\
\hline aaST14 $(n=13)$ & Ser & - & - & - & - & - & - & - & - & - & Gly & Thr & Asp & - & - & - & - & CPV-2b \\
\hline aaST15 $(n=2)$ & - & - & - & - & - & - & - & - & - & - & - & - & Glu & Ile & - & - & - & CPV-2C \\
\hline aaST16 $(n=1)$ & - & - & - & - & - & - & - & - & - & - & - & - & - & - & Ser & - & - & CPV-2a \\
\hline aaST17 $(n=1)$ & - & - & - & - & - & - & - & - & - & His & - & - & - & - & - & - & - & CPV-2a \\
\hline $\operatorname{aaST18}(n=1)$ & - & - & - & - & Ile & - & - & - & - & - & - & - & - & - & - & - & - & CPV-2a \\
\hline $\operatorname{aaST19}(n=1)$ & - & - & - & - & - & - & - & - & lle & - & - & - & - & - & - & - & - & CPV-2a \\
\hline aaST20 $(n=2)$ & - & - & - & - & Ile & - & - & - & - & - & - & - & Glu & - & - & - & - & CPV-2C \\
\hline $\operatorname{aaST21}(n=1)$ & - & - & - & - & - & - & - & - & - & - & Gly & Thr & Asp & - & - & - & - & $C P V-2 b$ \\
\hline
\end{tabular}

Amino acid positions are reported in the first line. In brackets: the number of nucleotide sequences belonging to the aaST. aaST amino acid sequence type, CPV- 2 canine parvovirus type 2

${ }^{a}$ CPV-2 antigenic variant deduced from amino acid residue 426

the CPV-2a and CPV-2b sequences appeared partially intermingled. The sequences corresponding to the most recent new CPV-2b variant (aaST14 and aaST21) formed a clearly distinguishable lineage in both phylogenetic trees and seemed to have an origin close to the CPV-2a viruses in the tree constructed with the nucleotide sequences (Fig. 2). A similar clustering was shown by the phylogenetic tree constructed on the VP2 amino acid sequence types obtained in this study and the worldwide reference sequences (Additional file 3). The aaSTs corresponding to the new CPV-2b appeared to be correlated to a Vietnamese CPV-2b variant identified in 2002 (AB120721) which, however, did not show the amino acid mutations $371 \mathrm{Ala} \rightarrow$ Gly and $418 \mathrm{Ile} \rightarrow$ Thr [30].

\section{Statistical analysis}

No statistical association was found among the CPV-2 antigenic variants, and sex, age, breed and vaccination status (Table 1). Only the year of sampling and geographical origin were statistically different between the CPV-2a, $2 \mathrm{~b}$ and $2 \mathrm{c}$ variants $(P<0.0001$ and $P=0.0001$, respectively). In the 24-year observation period, the frequency of the CPV-2 variants showed rapid oscillation (Fig. 3). The CPV-2a variant was commonly detected from 1994 to 2014, with the exception of 2013, and it was not found from 2015 to 2017. The CPV-2b variant was detected in 1995-1996, together with variant 2a; it was no longer detected from 1997 to 2008, and it reappeared in 2009. From 2009 until 2017, CPV-2b was

Table 4 Summaries of the sequence variability and Simpson's index of the three CPV-2 antigenic variants

\begin{tabular}{lllllllllll}
\hline & No. of sequences & Total no. of sites & $S$ & $\eta$ & $\pi$ & $\mathrm{s}$ & $\mathrm{a}$ & $\mathrm{a} / \mathrm{n}$ & $\mathrm{k}$ & $\mathrm{D}$ \\
\hline CPV-2a & 56 & 1745 & 53 & 55 & 0.00251 SD 0.00019 & 42 & 13 & 0.23636 & 4.384 & 0.02403 \\
CPV-2b & 18 & 1745 & 16 & 16 & 0.00227 SD 0.00061 & 11 & 5 & 0.3125 & 3.961 & 0.37255 \\
CPV-2c & 49 & 1745 & 26 & 26 & 0.00132 SD 0.00013 & 23 & 3 & 0.11538 & 2.301 & 0.08078 \\
Tot. & 123 & 1745 & 81 & 83 & 0.00329 SD 0.00012 & 63 & 20 & 0.24096 & 5.747 & 0.02519 \\
\hline
\end{tabular}

a: total number of non-synonymous differences. $a / \eta$ : total number of non-synonymous differences on the total number of mutations. CPV-2: canine parvovirus type 2. D: Simpson's index. k: average number of nucleotide differences. $\eta$ : total number of mutations. $\pi$ : nucleotide diversity (average number of nucleotide differences per site) and standard deviation. s: total number of synonymous differences. S: number of polymorphic (segregating) sites 


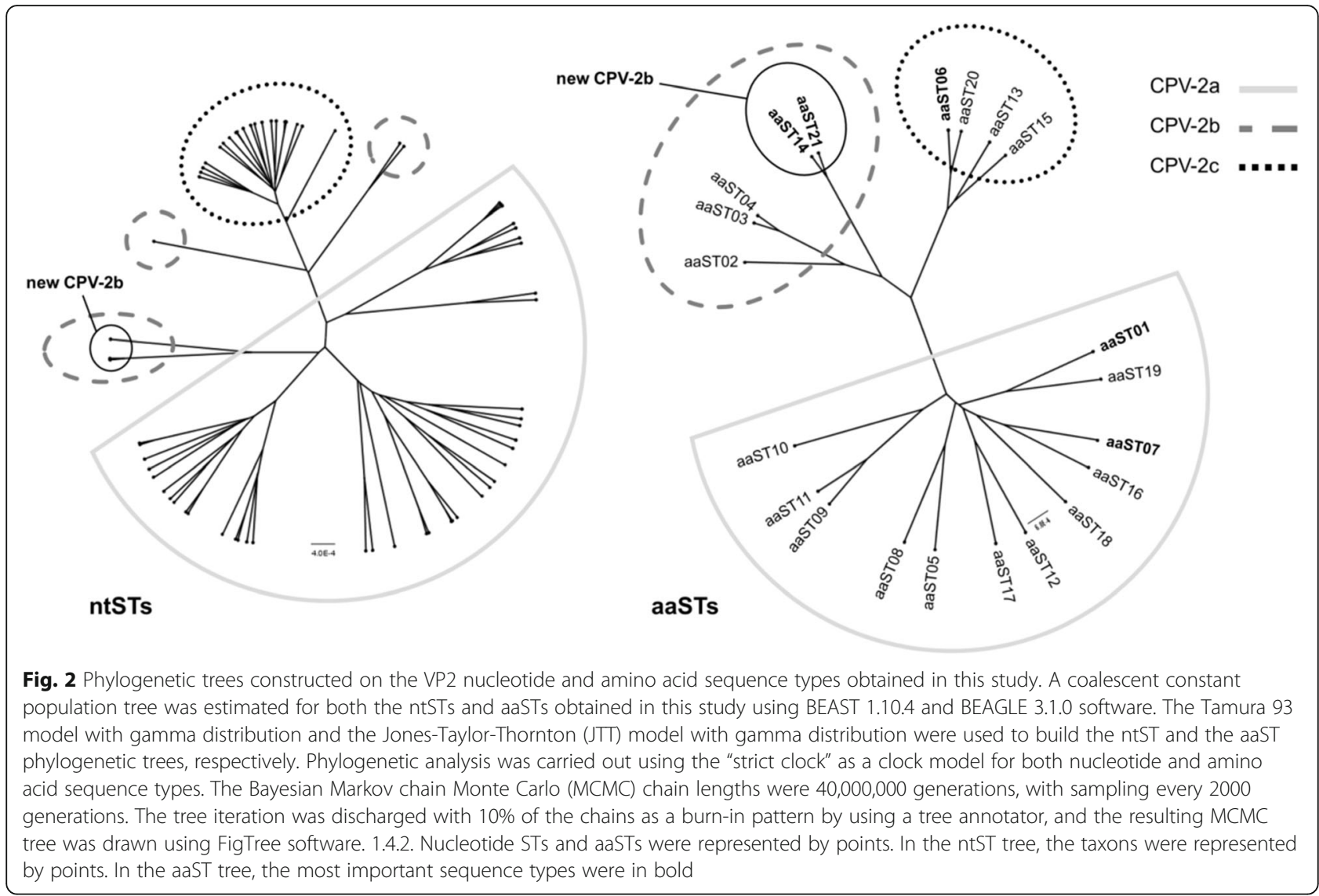

discovered, although not continuously. The CPV-2c variant was detected for the first time in 2000 and, subsequently, it was not continuously found until 2017 with a peak in 2009 represented by the strains from Sicily.

Regarding the distribution of the aaSTs over time, the majority of them appeared for just 1 year, at most 2 years, and were then no longer detected. Four aaSTs were an exception (Fig. 4): 1) aaST01, the predominant CPV-2a amino acid sequence type with 39/56 (66.1\%) nucleotide sequences, was detected from 1994 to 2014; 2) aaST07 (CPV-2a) was detected in 2000-2001, 20082009 and 2011 ; 3) aaST14, the predominant CPV-2b amino acid sequence type with 13/18 (72.2\%) nucleotide sequences, was detected for the first time in 2009 and it was also detected in 2010, 2013 and 2015-2017 and 4) aaST06, the predominant CPV-2c amino acid sequence type with $44 / 49$ (89.8\%) nucleotide sequences was detected for the first time in 2000 and it was not continuously detected until 2017 with a peak in 2009, represented by the Sicilian viruses.

\section{Discussion}

The present study provides an update regarding the distribution of the three CPV-2 variants in Italy from 1994 to 2017 and the evolution dynamics of the CPV-2 population in order to add new data regarding the diffusion and evolution of the three viral types $2 \mathrm{a}, 2 \mathrm{~b}$ and $2 \mathrm{c}$ over a long time frame. Although not all scientists are in agreement with the virus nomenclature, the use of the terms CPV-2a, $-2 \mathrm{~b}$ and $-2 \mathrm{c}$ is prevalent in the literature and, therefore, this terminology was used throughout the text.

During the 24-year study period, 123 diarrhoeic dogs testing positive for CPV-2 DNA were included in the study. For the most part, the dogs included were young (median age 3 months), reflecting the tropism of CPV-2 in dogs [3]. The majority of the dogs (76.4\%) came from the mainland of Italy and $23.6 \%$ of the dogs came from the island of Sicily in Southern Italy.

Approximately a third (32.5\%) of the dogs involved had been vaccinated, raising doubts regarding the correct application of the vaccination protocol or the ability of the vaccine to confer protective immunity. Previous studies had reported the occurrence of parvovirus infection in vaccinated dogs; the main cause of vaccination failure is the interfering role of maternal antibodies, although the evolution of the virus also raises questions regarding the efficacy of some vaccines and the necessity of updating the vaccine according to the new variants currently circulating in the canine population $[4,10,31,32]$. 


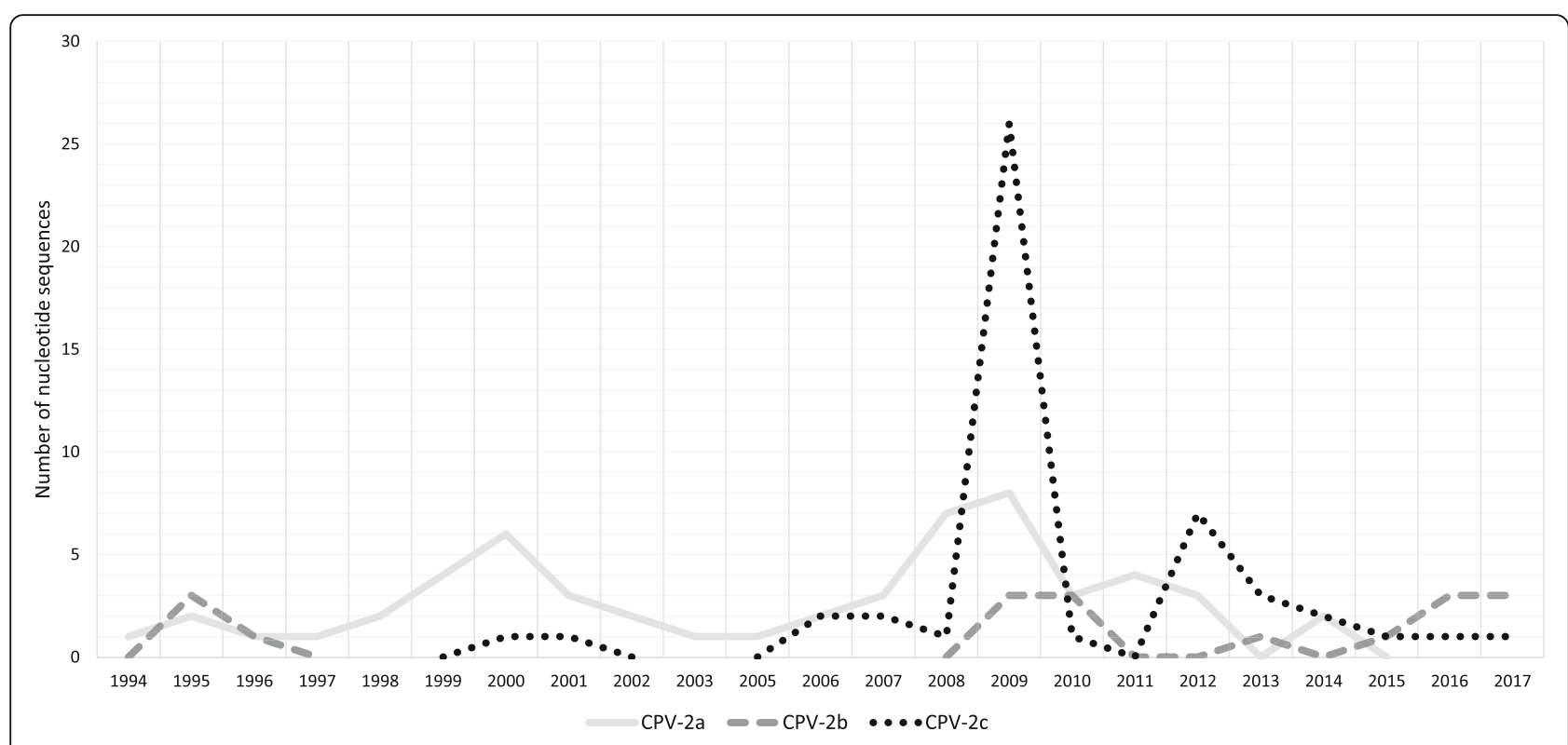

Fig. 3 Number of CPV-2 nucleotide sequences belonging to the CPV-2 antigenic variants identified in this study. CPV-2: canine parvovirus type 2

Other studies have demonstrated a high prevalence of non-responders, above all in certain breeds [33]. The vaccination protocol of the dogs tested in this study was unknown; however, since they were, for the most part, puppies, the potential cause of vaccination failure could have been interaction with maternally derived antibodies preventing the onset of effective immunity. Furthermore, pre-exposure to CPV-2 prior to vaccination could have been a contributing factor in some cases. No statistical relationship was found between the vaccination status,

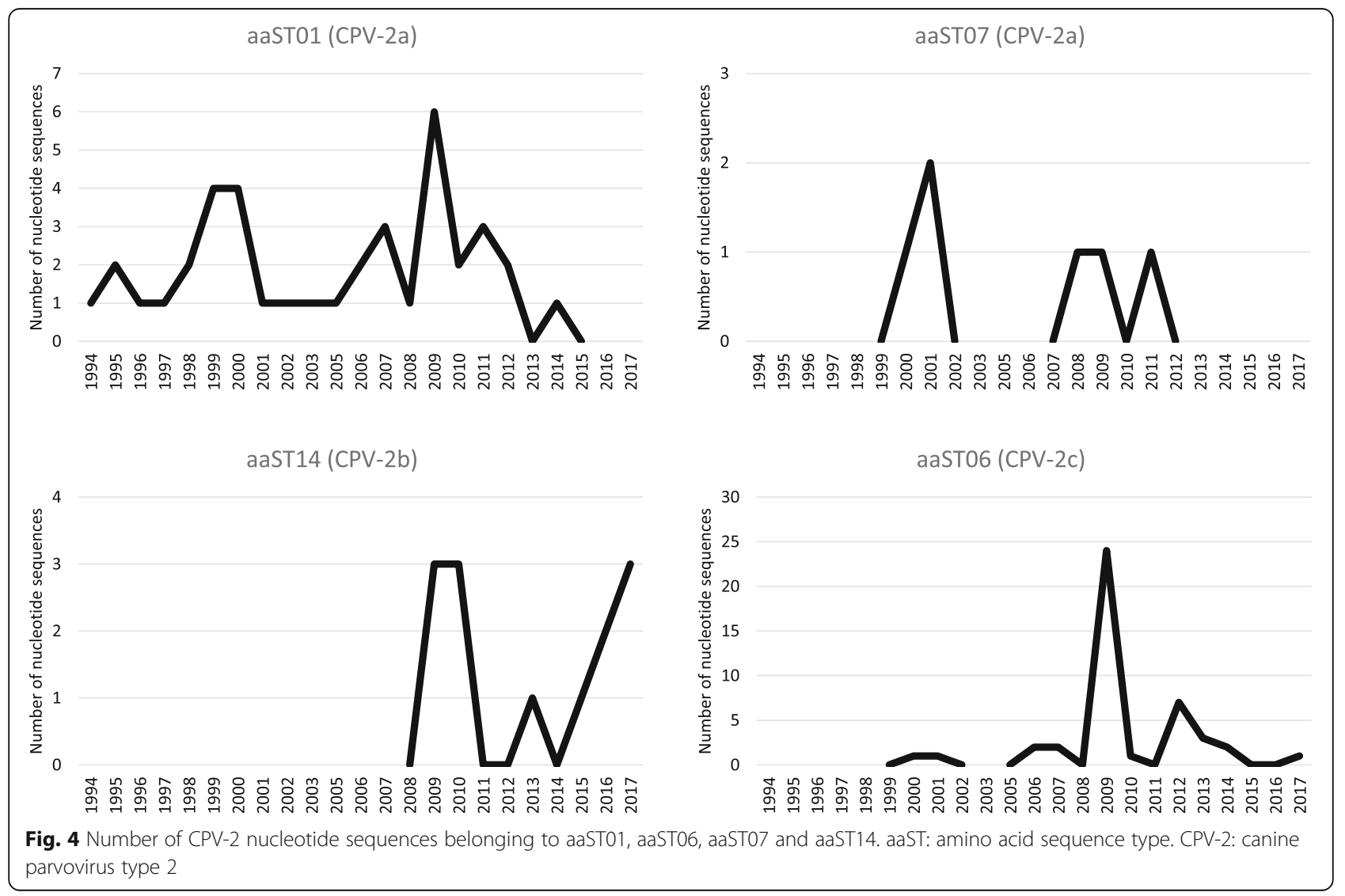


and the antigenic variants or aaSTs, excluding the fact that a specific antigenic variant or a specific amino acid sequence type may have eluded the vaccine-type immune response. A potential implication of the elevated evolution rate for vaccination efficacy was hypothesised. Canine parvovirus type 2 has the tendency of accumulating mutations, and the mismatch between vaccines and wild strains may have contributed to increasing the risk of an outbreak in the canine population; however, to date, a possible immune escape of CPV-2 variants has not yet been conclusively proven, and it has not been possible to reach any final conclusions $[34,35]$.

Canine parvovirus type $2 \mathrm{a}$ was the predominant variant detected in the dogs in this study, representing $45.5 \%$ of the viral sequences analysed, confirming previous data reported in Italy from 2008 to 2015 by Tucciarone and collaborators [15]. Canine parvovirus type 2a was continuously identified during the first 20 years of the study period, confirming its predominance in the Italian canine population until the first decade of the 2000s [13, 14]. This variant showed the highest diversity and sequence variability which was reflected by the largest number of ntSTs $(41 / 67,61.2 \%)$ and aaSTs $(12 / 21$, $57.1 \%)$ belonging to it. Furthermore, the Simpson diversity index, which is a measurement of diversity, was lower for type $2 \mathrm{a}$ than for the other CPV-2 variants. This result can allow hypothesising that the high diffusion in the canine population characterising the CPV-2a variant influenced the sequence variability of this variant, leading the virus to accumulate numerous nucleotide and amino acid mutations over time. In particular, of the 12 CPV-2a amino acid sequence types, aaST01 and aaST07 showed the highest fitness advantage. Amino acid ST01 was the most recurrent aaST, grouping 39 nucleotide sequences (25 ntSTs) identified from 1994 to 2014. Amino acid ST07, characterised by the amino acid mutation $440 \mathrm{Thr} \rightarrow$ Ala, grouped six nucleotide sequences (five ntSTs) detected in this study from 2000 to 2011. The 440 residue is located in the GH loop, a region exposed on the surface of the capsid and forms the $22 \AA$ threefold spike; the greatest variability between parvoviruses was observed in this antigenic region [36]. This mutation was found for the first time in Italy in 2000 in the 2a variant [27]; it was then reported worldwide. The recent CPV-2 variants detected in various parts of the world have the $440 \mathrm{Thr} \rightarrow$ Ala substitution; in several geographic areas, this change was restricted to the CPV-2c variant $[37,38]$ while, in other countries, it was also detected in both the CPV-2a and $2 b$ variants [39]. In this study, the $440 \mathrm{Thr} \rightarrow$ Ala change was limited to the CPV2a variant. In aaST16, which includes just one CPV-2a sequence (X83090/2009, GenBank ID: KF385388), the 440 $\mathrm{Thr} \rightarrow$ Ser change, which was previously described in one Italian FPV strain [40] and in Ecuadorian CPV-2 strains where it was shown in all variants $2 \mathrm{a}, 2 \mathrm{~b}$ and $2 \mathrm{c}$ [41] was detected. Another amino acid change involving residue 440 ( $\mathrm{Thr} \rightarrow$ Pro) has recently been reported in Sardinia, Italy [19], confirming the high variability of the region as well as the fact that residue 440 was under positive selective pressure.

The CPV-2b variant, detected in 18/123 dogs sampled from the mainland of Italy, was the least prevalent variant identified in this study. It was detected in the first years of this study but it was no longer detected between 1997 and 2008. Starting from 2009, CPV-2b was identified until 2017 in the mainland of Italy. This reappearance such a long time after its last detection, associated with its permanence in the canine population in subsequent years, could suggest that a new CPV-2b emerged. In fact, in 2009, a CPV-2b variant with a unique VP2 amino acid sequence corresponding to aaST14 was identified. Canine parvovirus type $2 \mathrm{~b}$ aaST14 was characterised by the amino acid mutations 13 Pro $\rightarrow$ Ser, 371 Ala $\rightarrow$ Gly and $418 \mathrm{Ile} \rightarrow$ Thr in the VP2 deduced protein. Amino acid ST14 grouped 13 nucleotide sequences (2 ntSTs), and it was the only CPV-2b aaST identified in this study from 2009 to 2017 with the exception of one CPV-2b variant detected in 2016 which alone constituted aaST21. The VP2 protein sequence of aaST21 was identical to aaST14 except for the lack of a mutation in position 13. Canine parvovirus type $2 \mathrm{~b}$ strains with analogous amino acid changes were detected in several parts of Italy during the same period (2008-2015) [15, 19], but they were not reported in other parts of the world. The amino acid mutations in residues 371 and 418 of the VP2 protein probably gave an adaptive advantage to the "new CPV-2b" and allowed it to spread and stabilise in the canine population. The 418 Ile $\rightarrow$ Thr substitution located in the GH loop of VP2 was reported for CPV$2 \mathrm{a}$ in Italy [21] and, in addition to Italy, it was also reported in Asian countries where it was a common mutation shown by Korean and Japanese strains [42, 43]. Additional studies are needed to understand how the amino acid mutations $371 \mathrm{Ala} \rightarrow$ Gly and $418 \mathrm{Ile} \rightarrow \mathrm{Thr}$ could affect the replicative efficiency of this virus, and whether this "new CPV-2b" originated from adaptive mutations of viruses already present in the Italian territory or whether it could have been introduced into the Italian territory by means of dogs being transported. Since, to date, this "new CPV-2b" has been detected only in Italy, it is probably the result of local differentiation. From the sequence analysis, CPV-2b was more genetically stable than CPV-2a but showed the highest fraction of non-synonymous mutations, highlighting a significant phenotypic effect of the accumulated mutations over time. A similar situation was observed in Australia where CPV-2b subtypes have continued to evolve at a relatively rapid rate in recent years [11]. 
The number of viruses belonging to the CPV-2c variant identified in this study (49/123, 39.8\%) was comparable to that of CPV-2a. This variant was detected for the first time in 2000 and was not continuously detected until the end of the study period. Nevertheless, CPV-2c appeared to be the least variable variant with only four aaSTs grouping $20 \mathrm{ntSTs}$ of which 17 were included in aaST06. The low variability of this variant was confirmed by the high number of nucleotide sequences $(44 / 49)$ referring to aaST06, identified from 2000 to 2017, both on the mainland and in insular areas, which therefore proved to have a high fitness in replicating and spreading in the canine population under study. Half of the CPV-2c sequences analysed (24/49) were sampled in Sicily in 2009, and 22 belonged to aaST06. From these data, it could be interpreted that the elevated number of homogeneous viral sequences coming from Sicily in 2009 may have altered the calculated variability of the $\mathrm{CPV}-2 \mathrm{c}$ variant. Instead, excluding all the viral sequences identified in dogs from Sicily, the CPV-2c variant was still the least variable. This was probably because aaST06 was highly widespread and also predominant on the mainland.

In Sicily, CPV-2c was the predominant variant (24/29), followed by CPV-2a with five identified viruses; CPV-2b was apparently not spread in dogs of the insular territory. The Sicilian viral strains analysed in the present study were collected in 2009-2010 while CPV-2b reappeared on the mainland of Italy in 2009; on the basis of this trend, the subsequent introduction and spread of CPV-2b in Sicily was conceivable. The latter hypothesis has to be excluded because a recent survey carried out in Sicily from 2009 to 2015 reported a high prevalence of the 2c variant in the absence of CPV-2b [18]. This significant difference between Sicily and the Italian mainland probably depended on the different geographical basis, illustrating different variant/subtypes among the areas analysed within the same country. Similarly, in Sardinia, the other major Italian island, a recent survey has shown an additional diverse epidemiological situation with a prevalence of CPV-2b [19].

From the data analysed, a representative aaST grouping the majority of the ntSTs was found for each CPV-2 antigenic variant. For CPV-2a, the predominant aaST was number 01; for CPV-2b, it was aaST14 and, for CPV-2c, it was aaST06, both on the mainland and in the insular areas of Italy. Taken together, these three aaSTs grouped 44/67 ntSTs and 96/123 VP2 nucleotide sequences identified in this study. The viruses showing the predominant amino acid sequence would probably be more adapted to replicate in the host and fit the environmental conditions, allowing them to spread and last over time in the canine population. All other aaSTs, being less adapted to spreading in the canine population, disappeared quickly after their emergence. In fact, with the exception of CPV-2a aaST07 grouped five ntSTs and six nucleotide sequences detected from 2000 to 2011; the remaining 17/21 aaSTs grouped 21/123 nucleotide sequences belonging to $18 / 67 \mathrm{ntSTs}$ and were identified for no more than 1 or 2 years in this study.

Sequence analysis revealed several coding changes, some of them involving residues located on the capsid surface, where important antigenic sites are located. Single mutations have been shown to affect the binding of monoclonal antibodies with consequent CPV-2 evasion of humoral immunity [44]. The high variability of the surface antigenic sites may also be responsible for variation in host specificity as indicated by the overlap of the transferrin receptor (TfR) binding site with the antigenic sites [45]. Although the functional impact of the majority of mutations has yet to be ascertained, the present study indicated active evolution of these strains.

\section{Conclusions}

From the data herein analysed, CPV-2a was the prevalent variant circulating in the canine population of mainland Italy from 1994 to the first decade of the 2000s. On the contrary, CPV-2b and 2c were not detected or were detected only sporadically until the 2000s, and have been successively identified with increased frequency. The CPV-2a variant was characterised by the highest genetic variability; instead, CPV-2c was characterised by notable stability with a predominant amino acid profile. Finally, from its remergence in recent years, CPV-2b has shown a new and distinctive profile of the VP2 protein, characterised by Asn 517-to-Ser and Ala 371-to-Gly changes. Additional studies are warranted to confirm an increased diffusion of the variants CPV-2b and $2 c$ at the expense of the CPV-2a on the Italian mainland and in many other countries [11,37]; monitoring of the evolution and spread of the new CPV-2 variants should be a key aim of ongoing research.

\section{Methods \\ Samples}

One-hundred and twenty-three diarrhoeic dogs which tested positive to CPV-2 DNA using a qualitative polymerase chain reaction (PCR) assay [46] carried out on faecal samples collected from 1994 to 2017 were retrospectively included in this study. Faecal samples were tested at the Service of Clinical Pathology (Department of Veterinary Medical Sciences - DIMEVET, University of Bologna, Italy) and at the Istituto Zooprofilattico Sperimentale della Sicilia "A. Mirri" (Palermo, Italy). Signalment data (year of sampling, sex, age, breed and geographical origin) and vaccination status of the dogs which tested positive were retrieved from medical records. 


\section{Molecular characterisation of the CPV-2 strains}

Viral DNA was extracted from faecal samples using the NucleoSpin Tissue Mini Kit (Macherey-Nagel, Düren, Germany) according to the manufacturer's instructions. The VP2 gene of CPV-2 was amplified as previously described by Battilani and collaborators [47], and amplicons of expected size (1745 nts) were directly sequenced using the Sanger method, using both forward and reverse primers, and a third internal primer, primer 41 (5' - GCC CTT GTG TAG ACG C -3') [48].

The nucleotide sequences obtained were aligned using the CLUSTAL W method implemented in BioEdit sequence alignment editor version 7.2.5 [49]. and were translated into amino acid sequences. The antigenic variants (CPV-2a, 2b and 2c) were deduced from the sequences based on amino acid residue 426. The sequences were grouped on the basis of nucleotide heterogeneity into different ntSTs which shared unique genotypes and on the basis of amino acid heterogeneity in different aaSTs which shared unique deduced protein sequences. The ntSTs and aaSTs obtained were compared to 175 worldwide nonItalian CPV-2 reference sequences (Additional file 4) available from the GenBank database (https://www.ncbi.nlm. nih.gov/genbank/), and the degree of similarity among the sequences at both the nucleotide and the amino acid levels was determined using BioEdit software.

In order to estimate the genetic diversity and the sequence variability of the sequence data, a variety of statistical analyses regarding nucleotide polymorphism and sequence variability were assessed using DnaSP version 6 [50]. Statistical analysis was carried out on the antigenic variant (CPV-2a, $2 \mathrm{~b}$ and $2 \mathrm{c})$ subpopulations. The following parameters were estimated: the number of polymorphic sites $(S), \pi$ (which was the average number of nucleotide differences per site between two sequences) and its standard deviation, the total number of mutations ( $\eta$ ), the total number of synonymous (s) and non-synonymous (a) differences, the $\mathrm{a} / \eta$ rate and $\mathrm{k}$. In addition, $\mathrm{D}$ was calculated as a measure of population diversity:

$$
\mathrm{D}=\frac{\sum \mathrm{n}(\mathrm{n}-1)}{\mathrm{N}(\mathrm{N}-1)}
$$

where $\mathrm{N}$ is the total number of all sequences and $\mathrm{n}$ is the number of sequences for each nucleotide sequence type (ntST). The value of D ranged from 0 to 1 . A D value of 0 represented infinite diversity and 1 corresponded to no diversity, that is, the lower the value of $D$, the greater the species diversity [51].

\section{Phylogenetic analysis}

The viral sequences obtained in this study were analysed using BEAST version 1.10.4 and BEAGLE version 3.1.0 software [52-54] using a strict clock model with a coalescent constant population. Tamura-Nei and JonesTaylor-Thornton (JTT) models with gamma distribution were used to build the ntST and the aaST phylogenetic trees, respectively. In addition, a set of protein sequences including 175 worldwide non-Italian CPV-2 amino acid reference sequences (Additional file 4) was analysed using a strict clock model with a coalescent constant population and a Jones-Taylor-Thorton (JTT) model with gamma distribution. The Bayesian Markov chain Monte Carlo (MCMC) chain lengths were 40,000,000 generations, with sampling every 2000 generations. The tree iteration was discharged with $10 \%$ of the chains as a burn-in pattern by using a tree annotator, and the resulting MCMC tree was drawn with FigTree software (v1.4.2) (http://tree.bio.ed.ac.uk/software/figtree/).

\section{Statistical analysis}

Signalment data and vaccination status were grouped as reported in Table 1 and were evaluated using standard descriptive statistics. In particular, the distribution of the three CPV-2 antigenic variants and the aaSTs detected during the 24-year sampling period were compared. The viruses detected were grouped on the basis of the antigenic variant (CPV-2a, $2 \mathrm{~b}$ and $2 \mathrm{c})$, and clinical data were analysed using the Chi-squared $\left(\chi^{2}\right)$ test. The results were considered significant when $\mathrm{P}$ was $<0.05$. Statistical analyses were carried out using statistical software (MedCalc Statistical Software version 18.5, MedCalc Software bvba, Ostend, Belgium).

\section{Supplementary information}

Supplementary information accompanies this paper at https://doi.org/10. 1186/s12917-019-2096-1.

Additional file 1. Canine parvovirus type 2 (CPV-2) antigenic variants, amino acid sequence types (aaSTs), nucleotide sequence types (ntSTs) and nucleotide sequences obtained in this study. Sequences are reported with: year of identification, lab ID number, antigenic variant and GenBank accession number

Additional file $\mathbf{2}$ Sequence variability and Simpson's index of the canine parvovirus type $2 c(C P V-2 c)$ sequences without Sicilian strains and of the CPV-2c Sicilian sequences. a: total number of non-synonymous differences. $\mathrm{a} / \mathrm{n}$ : total number of non-synonymous differences on the total number of mutations. CPV-2: canine parvovirus type 2. D: Simpson's index. k: average number of nucleotide differences. $\eta$ : total number of mutations. $\pi$ : nucleotide diversity (average number of nucleotide differences per site) and standard deviation. s: total number of synonymous differences. S: number of polymorphic (segregating) sites.

Additional file 3. The unrooted phylogenetic tree constructed on VP2 amino acid sequence types obtained in this study and worldwide nonItalian canine parvovirus type 2 (CPV-2) amino acid reference sequences. A coalescent constant population tree was estimated using BEAST 1.10.4 and BEAGLE 3.1.0 software utilising a strict clock model with a coalescent constant population. The Jones-Taylor-Thornton (JTT) model with gamma distribution was used to build the phylogenetic trees. Phylogenetic analysis was carried out using the "strict clock" as a clock model; 175 worldwide non-Italian CPV-2 amino acid reference sequences (Additional file 4) were included in the analysis. The Bayesian Markov chain Monte Carlo (MCMC) chain lengths were 40,000,000 generations, with sampling 
every 2000 generations. The tree iteration was discharged with $10 \%$ of the chains as a burn-in pattern by using a tree annotator and the resulting MCMC tree was drawn using FigTree software 1.4.2. In blue, the CPV-2a aaSTs obtained in this study. In red, the CPV-2b aaSTs obtained in this study. In green the CPV-2C aaSTs obtained in this study.

Additional file 4. List of the 175 worldwide non-Italian canine parvovirus type 2 (CPV-2) reference sequences retrieved from the GenBank database and used for sequence analysis. Sequences are reported with: year of identification, acronym of the nation, antigenic variant and GenBank accession number.

\section{Abbreviations}

a: Total number of non-synonymous differences; $a / n$ : Total number of nonsynonymous differences on the total number of mutations; aaST: Amino acid sequence type; Ala: Alanine; Arg: Arginine; Asn: Asparagine; Asp: Aspartic acid; CPV-2: Canine parvovirus type 2; D: Simpson's index; FPV: Feline panleukopenia virus; Glu: Glutamic acid; Gly: Glycine; His: Histidine; Ile: Isoleucine; JTT: Jones-Taylor-Thornton; k: Average number of nucleotide differences; Lys: Lysine; MCMC: Markov chain Monte Carlo; MEV: Mink enteritis virus; nts: Nucleotides; ntST: Nucleotide sequence type PCR: Polymerase chain reaction; Pro: Proline; RaPV: Raccoon parvovirus: $S$ : Number of polymorphic sites; s: Total number of synonymous differences; Ser: Serine; Thr: Threonine; Val: Valine; VP2: Viral protein 2; $\eta$ : Total number of mutations; $\pi$ : Nucleotide diversity; $\times 2$ : Chi-squared

\section{Acknowledgements}

None

\section{Authors' contributions}

$M B, A B$ and $A G$ designed the study. MB, AB, FMi and GP collected the faecal samples and the background information on the samples. AB, FMo, FMi and SDB carried out CPV-2 molecular detection and VP2 sequencing. MB, FMo and $A B$ analysed the sequence data. $A B$ carried out the statistical analyses. $M B, A B$ and $F M o$ drafted the manuscript. FMi, GP, SDB and AG critically revised the manuscript for important intellectual content. All the authors have read the manuscript and approved the final version.

\section{Funding}

This study received no grant from any funding agency in the public or commercial sectors.

\section{Availability of data and materials}

The datasets supporting the conclusions of this article are included within the article (and its additional files). The nucleotide sequences generated during the current study are available in the GenBank repository under accession numbers: KF373568-KF373611, KF385382-KF385391 and MK348065MK348125. The sequences AF306445-AF306450, AF393506 and AF401519 originated in previous studies $[13,27]$.

\section{Ethics approval and consent to participate}

The study was carried out using stored faecal samples which had been collected with the agreement of the dog owners for clinical and laboratory purposes independent of the study. As stored faecal samples were used, no separate ethical approval was required for the study. All efforts were made to minimise the discomfort of the animals during sampling.

\section{Consent for publication}

Not applicable.

\section{Competing interests}

The authors declare that they have no competing interests.

\section{Author details}

'Department of Veterinary Medical Sciences, Alma Mater Studiorum University of Bologna, Via Tolara di Sopra 50, 40064 Ozzano dell'Emilia, BO, Italy. ${ }^{2}$ Istituto Zooprofilattico Sperimentale della Sicilia "A. Mirri", Palermo, taly
Received: 6 June 2019 Accepted: 16 September 2019

Published online: 04 November 2019

\section{References}

1. International Committee on Taxonomy of Viruses ICTV. http://ictvonline.org/ virustaxonomy.asp. Accessed 10 Apr 2019.

2. Cotmore SF, Agbandje-McKenna M, Chiorini JA, Mukha DV, Pintel DJ, Qiu J, Soderlund-Venermo M, Tattersall P, Tijssen P, Gatherer D, Davison AJ. The family Parvoviridae. Arch Virol. 2014;159:1239-47.

3. Greene CE, Decaro N. Canine viral enteritis. In: Greene CE, editor. Infectious diseases of the dog and cat. 4th ed. USA: Elsevier; 2012. p. 67-75.

4. Decaro N, Cirone F, Desario C, Elia G, Lorusso E, Colaianni ML, Martella V, Buonavoglia C. Severe parvovirus in a 12-year-old dog that had been repeatedly vaccinated. Vet Rec. 2009;164:593-5.

5. Shackelton LA, Parrish CR, Truyen U, Holmes EC. High rate of viral evolution associated with the emergence of carnivore parvovirus. Proc Natl Acad Sci U S A. 2005;102:379-84.

6. Parrish CR, Aquadro CF, Strassheim ML, Evermann JF, Sgro J, Mohammed HO. Rapid antigenic-type replacement and DNA sequence evolution on canine parvovirus. J Virol. 1991:65:6544-52.

7. Buonavoglia C, Martella V, Pratelli A, Tempesta M, Cavalli A, Buonavoglia D, Bozzo G, Elia G, Decaro N, Carmichael LE. Evidence for evolution of canine parvovirus type-2 in Italy. J Gen Virol. 2001;82:3021-5.

8. Truyen U, Evermann JF, Vieler E, Parrish CR. Evolution of canine parvovirus involved loss and gain of feline host range. Virology. 1996;215:186-9.

9. Decaro N, Buonavoglia C. Canine parvovirus-a review of epidemiological and diagnostic aspects, with emphasis on type 2c. Vet Microbiol. 2012;155: $1-12$.

10. Miranda C, Thompson G. Canine parvovirus: the worldwide occurrence of antigenic variants. J Gen Virol. 2016:97:2043-57.

11. Clark NJ, Seddon JM, Kyaw-Tanner M, Al-Alawneh J, Harper G, McDonagh P, Meers J. Emergence of canine parvovirus subtype $2 \mathrm{~b}$ (CPV-2b) infections in Australian dogs. Infect Genet Evol. 2018:58:50-5.

12. Woolford L, Crocker P, Bobrowski H, Baker T, Hemmatzadeh F. Detection of the canine parvovirus 2c subtype in Australian dogs. Viral Immunol. 2017;30: $371-6$.

13. Battilani M, Scagliarini A, Tisato E, Turilli C, Jacoboni I, Casadio R, Prosperi S. Analysis of canine parvovirus sequences from wolves and dog isolated in taly. J Gen Virol. 2001;82:1555-60.

14. Decaro N, Desario C, Addie DD, Martella V, Vieira MJ, Elia G, Zicola A, Davis C, Thompson G, Thiry E, Truyen U, Buonavoglia C. The study molecular epidemiology of canine parvovirus, Europe. Emerg Infect Dis. 2007;13:1222-4.

15. Tucciarone CM, Franzo G, Mazzetto E, Legnardi M, Caldin M, Furlanello T, Cecchinato M, Drigo M. Molecular insight into Italian canine parvovirus heterogeneity and comparison with the worldwide scenario. Infect Genet Evol. 2018:66:171-9.

16. Decaro N, Elia G, Martella V, Campolo M, Desario C, Camero M, Cirone F, Lorusso E, Lucente MS, Narcisi D, Scalia P, Buonavoglia C. Characterisation of the canine parvovirus type 2 variants using minor groove binder probe technology. J Virol Methods. 2006:133:92-9.

17. Cavalli A, Martella V, Elia G, Buonavoglia D, Pratelli A, Decaro N, Buonavoglia C, Carmichael L, Bozzo G, Tempesta M. Evidence for evolution of canine parvovirus type 2 in Italy. J Gen Virol. 2001;82:3021-5.

18. Purpari G, Mira F, Di Bella S, Di Pietro S, Giudice E, Guercio A. Investigation on canine parvovirus circulation in dogs from Sicily (Italy) by biomolecular assay. Acta Vet (Beogr). 2018;68:80-94

19. Dei Giudici S, Cubeddu T, Giagu A, Sanna G, Rocca S, Oggiano A. First molecular characterization of canine parvovirus strains in Sardinia, Italy. Arch Virol. 2017:162:3481-6.

20. Ohshima T, Hisaka M, Kawakami K, Kishi M, Tohya Y, Mochizuki M Chronological analysis of canine parvovirus type 2 isolates in Japan. J Vet Med Sci. 2008;70:769-75

21. Decaro N, Desario C, Parisi A, Martella V, Lorusso A, Miccolupo A, Mari V, Colaianni ML, Cavalli A, Di Trani L, Buonavoglia C. Genetic analysis of canine parvovirus type 2c. Virology. 2009;385:5-10.

22. Ikeda Y, Mochizuki M, Naito R, Nakamura K, Miyazawa T, Mikami T, Takahashi E. Predominance of canine parvovirus (CPV) in unvaccinated cat populations and emergence of new antigenic types of CPVs in cats. Virology. 2000;278: 13-9.

23. Zhou $P$, Zeng $W$, Zhang $X$, Li S. The genetic evolution of canine parvovirus a new perspective. PLoS One. 2017;12:e0175035 
24. Maya L, Calleros L, Francia L, Hernández M, Iraola G, Panzera Y, Sosa K, Pérez R. Phylodynamics analysis of canine parvovirus in Uruguay: evidence of two successive invasions by different variants. Arch Virol. 2013;158:1133-41.

25. Hoelzer K, Shackelton LA, Parrish CR, Holmes EC. Phylogenetic analysis reveals the emergence, evolution and dispersal of carnivore parvoviruses. J Gen Virol. 2008:89:2280-9.

26. Hoelzer K, Parrish CR. The emergence of parvoviruses of carnivores. Vet Res. 2010;41:39.

27. Battilani M, Ciulli S, Tisato E, Prosperi S. Genetic analysis of canine parvovirus isolates (CPV-2) from dogs in Italy. Virus Res. 2002;83:149-57.

28. Battilani M, Balboni A, Ustulin M, Giunti M, Scagliarini A, Prosperi S. Genetic complexity and multiple infections with more parvovirus species in naturally infected cats. Vet Res. 2011;42:43.

29. Allison AB, Organtini $\sqcup$, Zhang S, Hafenstein SL, Holmes EC, Parrish CR. Single mutations in the VP2 300 loop region of the three-fold spike of the carnivore parvovirus capsid can determine host range. J Virol. 2016;90:753-67.

30. Nakamura M, Tohya Y, Miyazawa T, Mochizuki M, Phung HT, Nguyen NH, Huynh LM, Nguyen LT, Nguyen PN, Nguyen PV, Nguyen NP, Akashi H. A novel antigenic variant of canine parvovirus from a Vietnamese dog. Arch Virol. 2004;149:2261-9.

31. Decaro N, Desario C, Elia G, Campolo M, Lorusso A, Mari V, Martella V, Buonavoglia C. Occurrence of severe gastroenteritis in pups after canine parvovirus vaccine administration: a clinical and laboratory diagnostic dilemma. Vaccine. 2007;25:1161-6.

32. Mittal M, Chakravarti S, Mohapatra JK, Chug PK, Dubey R, Upmanuyu V, Narwal PS, Kumar A, Churamani CP, Kanwar NS. Molecular typing of canine parvovirus strains circulating from 2008 to 2012 in an organized kennel in India reveals the possibility of vaccination failure. Infect Genet Evol. 2014;23: $1-6$.

33. Mira F, Dowgier G, Purpari G, Vicari D, Di Bella S, Macaluso G, Gucciardi F, Randazzo V, Decaro N, Guercio A. Molecular typing of a novel canine parvovirus type 2a mutant circulating in Italy. Infect Genet Evol. 2018;61:67-73.

34. Truyen U. Evolution of canine parvovirus - a need for new vaccines? Vet Microbiol. 2006:117:9-13.

35. Hernández-Blanco B, Catala-Lópeza F. Are licensed canine parvovirus (CPV2 and (PV2b) vaccines able to elicit protection against CPV2c subtype in puppies?: a systematic review of controlled clinical trials. Vet Microbiol. 2015;80:1-9.

36. Chapman MS, Rossmann RG. Structure, sequence, and function correlations among parvoviruses. Virology. 1993:194:491-508.

37. Calderón MG, Romanutti C, D'Antuono A, Keller L, Mattion N, La Torre J. Evolution of canine parvovirus in Argentina between years 2003 and 2010: CPV2C has become the predominant variant affecting the domestic dog population. Virus Res. 2011;157:106-10.

38. Kapil S, Cooper E, Lamm C, Murray B, Rezabek G, Johnston L 3rd, Campbell $\mathrm{G}$, Johnson B. Canine parvovirus types $2 \mathrm{c}$ and $2 \mathrm{~b}$ circulating in north American dogs in 2006 and 2007. J Clin Microbiol. 2007:45:4044-7.

39. Clegg SR, Coyne KP, Parker J, Dawson S, Godsall SA, Pinchbeck G, Cripps PJ, Gaskell RM, Radford AD. Molecular epidemiology and phylogeny reveal complex spatial dynamics in areas where canine parvovirus is endemic. J Virol. 2011:85:7892-9.

40. Decaro N, Desario C, Miccolupo A, Campolo M, Parisi A, Martella V, Amorisco F, Lucente MS, Lavazza A, Buonavoglia C. Genetic analysis of feline panleukopenia viruses from cats with gastroenteritis. J Gen Virol. 2008;89: 2290-8

41. Aldaz J, Garcıa-Dıaz J, Calleros L, Katia S, Gregorio I, Marandino A, Hernàndez M, Panzera Y, Pérez R. High local genetic diversity of canine parvovirus from Ecuador. Vet Microbiol. 2013;166:214-9.

42. Jeoung SY, Ahn SJ, Kim D. Genetic analysis of VP2 gene of canine parvovirus isolates in Korea. J Vet Med Sci. 2008;70:719-22.

43. Soma T, Taharaguchi S, Ohinata T, Ishii H, Hara M. Analysis of the VP2 protein gene of canine parvovirus strains from affected dogs in Japan. Res Vet Sci. 2013;94:368-71.

44. Chang SF, Sgro JY, Parrish CR. Multiple amino acids in the capsid structure of canine parvovirus coordinately determine the canine host range and specific antigenic and hemagglutination properties. J Virol. 1992;66:6858-67.

45. Hafenstein S, Bowman VD, Sun T, Nelson CDS, Palermo LM, Chipman PR, Battisti AJ, Parrish CR, Rossmann MG. Structural comparison of different antibodies interacting with parvovirus capsids. J Virol. 2009;83:5556-66.
46. Mochizuki M, San Gabriel MC, Nakatani H, Yoshida M. Comparison of polymerase chain reaction and haemagglutination assays for the detection of canine parvoviruses in faecal specimens. Res Vet Sci. 1993;55:60-3.

47. Battilani M, Scagliarini A, Ciulli S, Morganti L, Prosperi S. High genetic diversity of the VP2 of a canine parvovirus strain detected in a domestic cat. Virology. 2006;352:22-6.

48. Truyen U, Müller T, Heidrich R, Tackmann K, Carmichael LE. Survey on viral pathogens in wild red foxes (Vulpes vulpes) in Germany with emphasis on parvoviruses and analysis of a DNA sequence from a red fox parvovirus. Epidemiol Infect. 1998;121:433-40.

49. Hall TA. BioEdit: a user-friendly biological sequence alignment editor and analysis program for windows 95/98/NT. Nucl Acids Symp Ser. 1999;41:95-8.

50. Rozas J, Ferrer-Mata A, Sánchez-DelBarrio JC, Guirao-Rico S, Librado P, Ramos-Onsins SE, Sánchez-Gracia A. DnaSP 6: DNA sequence polymorphism analysis of large datasets. Mol Biol Evol. 2017:34:3299-302.

51. Simpson EH. Measurement of diversity. Nature. 1949;163:688.

52. Ayres DL, Darling A, Zwickl DJ, Beerli P, Holder MT, Lewis PO, Huelsenbeck JP, Ronquist F, Swofford DL, Cummings MP, Rambaut A, Suchard MA. BEAGLE: an application programming interface and high-performance computing library for statistical phylogenetics. Syst Biol. 2012;61:170-3.

53. Ayres DL, Cummings MP, Baele G, Darling AE, Lewis PO, Swofford DL, Huelsenbeck JP, Lemey P, Rambaut A, Suchard MA. BEAGLE 3: improved performance, scaling, and usability for a high-performance computing library for statistical phylogenetics. Syst Biol. 2019. https://doi.org/10.1093/ sysbio/syz020

54. Suchard MA, Lemey P, Baele G, Ayres DL, Drummond AJ, Rambaut A. Bayesian phylogenetic and phylodynamic data integration using BEAST 1. 10. Virus Evol. 2018;4:vey016.

\section{Publisher's Note}

Springer Nature remains neutral with regard to jurisdictional claims in published maps and institutional affiliations.

Ready to submit your research? Choose BMC and benefit from:

- fast, convenient online submission

- thorough peer review by experienced researchers in your field

- rapid publication on acceptance

- support for research data, including large and complex data types

- gold Open Access which fosters wider collaboration and increased citations

- maximum visibility for your research: over $100 \mathrm{M}$ website views per year

At $\mathrm{BMC}$, research is always in progress.

Learn more biomedcentral.com/submissions 